\title{
Volumetric Description of Brain Atrophy in Neuronal Ceroid Lipofuscinosis 2: Supratentorial Gray Matter Shows Uniform Disease Progression
}

\author{
(D) U. Löbel, (D). Sedlacik, (D) M. Nickel, (D) S. Lezius, (D). Fiehler, Iㅣ. Nestrasil, (D)A. Kohlschütter, and (D) A. Schulz
}

\begin{abstract}
BACKGROUND AND PURPOSE: Experimental therapies for ceroid lipofuscinosis, neuronal, 2 (CLN2), a genetic disorder of childhood associated with progressive brain atrophy, are currently being developed. Because quantitative descriptions of the natural course of brain volume loss are needed to evaluate novel therapies, we performed MR imaging volumetry of patients with CLN2 to identify a suitable MR imaging marker of disease progression.
\end{abstract}

MATERIALS AND METHODS: Thirteen patients ( 8 females, 5 males) were recruited from a prospective natural disease cohort of patients with neuronal ceroid lipofuscinosis. Repeated MR imaging volumetric analysis (29 datasets) was performed by using the FreeSurfer Software Suite. Follow-up time ranged from 8 months to 5.3 years. MR imaging-segmented brain volumes were correlated to patient age and clinical scores.

RESULTS: Segmented brain volumes correlated significantly with patient age (lateral ventricles, $r=0.606, P=.001$; supratentorial cortical $\mathrm{GM}, r=-0.913, P<.001$; supratentorial WM, $r=-0.865, P<.001$; basal ganglia/thalamus, $r=-0.832, P<.001$; cerebellar GM, $r=-0.659$, $P<.001$; cerebellar WM, $r=-0.830, P<.001$ ) and clinical scores (lateral ventricles, $r=-0.692, P<.001$; supratentorial cortical GM, $r=$ $0.862, P<.001$; supratentorial WM, $r=0.735, P<.001$; basal ganglia/thalamus, $r=0.758, P<.001$; cerebellar GM, $r=0.609, P=.001$; cerebellar WM, $r=0.638, P<.001$ ). Notably, supratentorial cortical GM showed a uniform decline across the patient cohort. During late stages of the disease when the clinical score was zero, segmented brain volumes still correlated with patient age; this finding suggests that MR imaging volumetry allows quantitative assessment of disease progression at stages when it cannot be detected by clinical assessment alone.

CONCLUSIONS: Automated MR imaging volumetry, as a nonsubjective and highly sensitive tool, is feasible in CLN2 disease and provides a quantitative basis to evaluate novel experimental therapies.

ABBREVIATIONS: $C L N$ = ceroid lipofuscinosis, neuronal; $N C L=$ neuronal ceroid lipofuscinoses; $T$ PP1 $=$ tripeptidyl-peptidase-1

$\mathbf{N}$ euronal ceroid lipofuscinoses (NCL) are a group of neurodegenerative disorders with onset in childhood and are typically inherited in an autosomal recessive fashion. Their incidence

Received December 22, 2015; accepted after revision March 21, 2016.

From the Departments of Diagnostic and Interventional Neuroradiology (U.L., J.S., J.F.), Pediatrics (M.N., A.K., A.S.), and Medical Biometry and Epidemiology (S.L.), University Medical Center Hamburg-Eppendorf, Hamburg, Germany; and Department of Pediatrics (I.N.), University of Minnesota, Minneapolis, Minnesota.

The research leading to this publication received funding from the European Union Seventh Framework Program as part of the project "DEM-CHILD" (Grant agreement number 281234 to Angela Schulz) and from the German Federal Ministry of Education and Research (Grant NCL2Treat to Angela Schulz).

Paper previously presented at: European Society of Magnetic Resonance in Neuropediatrics, May 14-16, 2015; Porto, Portugal.

Please address correspondence to Ulrike Löbel, MD, Department of Diagnostic and Interventional Neuroradiology, O-22, Martinistr 52, 20246 Hamburg, Germany; e-mail: u.loebel@uke.de

- Indicates open access to non-subscribers at www.ajnr.org

$\equiv$ Indicates article with supplemental on-line table. in European countries ranges from 1:14,000 to $1: 100,000 .^{1}$ To date, at least 13 different genetic defects, ceroid lipofuscinosis neuronal (CLN) 1-14, are known. ${ }^{2}$ They form the basis of a novel classification system that includes the genetic defect and the time of disease onset (eg, CLN2, late-infantile). ${ }^{3}$

CLN2 is one of the most common forms of NCL (MIM No. 204500). It is caused by mutations in the CLN2 gene, which codes for the lysosomal enzyme tripeptidyl-peptidase- 1 (TPP1).TPP1 is a serine protease responsible for the breakdown of certain neuropeptides. A deficiency of TPP1 leads to an accumulation of lysosomal storage material in different cell types, including neurons, astrocytes, vascular endothelial and smooth-muscle cells, fibroblasts, adipocytes, and skeletal muscle. ${ }^{2}$ CLN2 disease subsequently leads to neuronal degeneration and brain atrophy. Patients with CLN2 have epilepsy and a decline in cognition,

http://dx.doi.org/10.3174/ajnr.A4816 
language, and gross motor function starting at 2-4 years of age. The disease rapidly progresses, with loss of vision due to optic atrophy and macular and retinal changes occurring in later disease stages. ${ }^{4}$ Nonclassic presentations (ie, juvenile, adult, or infantile onset) exist as well. ${ }^{3,5-8}$

On conventional MR imaging, a marked supratentorial and infratentorial atrophy with ventriculomegaly is the typical finding. In addition, a progressive-but-mild increase of WM signal intensity on T2WI and increased ADC values are observed., ${ }^{9,10}$ These findings have been largely related to myelin loss and gliosis $^{11}$ and contrast with the decreasing ADC values observed during normal brain maturation and myelination. ${ }^{12}$ Longitudinal MR imaging is rarely performed in patients with NCL because therapeutic options do not exist. Longitudinal MR brain volumetric studies have only been reported for CLN3 disease, ${ }^{13}$ and only 1 cross-sectional study in patients with CLN2, focusing on the size of CSF spaces, is available. ${ }^{14}$

The purpose of our study was to test our hypothesis that MR imaging brain volumetry provides a quantitative tool to assess the natural course of disease progression in CLN2 disease. More specifically, we hypothesized that GM volumes would be more strongly linked to the clinical disease course compared with WM regions and CSF spaces because CLN2 primarily involves the degeneration of neurons in the cerebral and cerebellar cortices. We aimed to establish reference values for current and future clinical studies (intraventricular enzyme replacement [NCT01907087], gene therapy [NCT01414985]; www.clinicaltrials.gov).

\section{MATERIALS AND METHODS \\ Patients}

Thirteen patients with confirmed defects of the CLN2 gene underwent MR imaging between 2008 and 2015 (On-line Table). Two patients are identical twins (457-1 and 457-2). Approval from the local ethics committee and written informed consent from the parents were obtained before inclusion of the patients into the study.

\section{Clinical Scoring}

The clinical course was assessed by using an established clinical rating scale for CLN2 disease. ${ }^{15}$ The scale represents a 12-point inventory of disease-based clinical assessments. The original scale consists of 4 functional domains: motor, language, vision, and tonic-clonic seizures. Within each domain, scores ranging from 0 to 3 are given, with 0 representing the absence of function and 3 , the age-appropriate normal function. The clinical score for tonicclonic seizures may be strongly influenced by the individual pharmacologic management of each patient and may show high variability. Therefore, it was not used in this study. To address the period before the diagnosis of CLN2 disease, we performed ratings retrospectively on the basis of patient charts and parent interviews. Once the diagnosis of CLN2 was established, ratings were obtained prospectively at 6-month intervals at the NCL specialty clinic in Hamburg. Three independent raters performed the clinical scoring, and the average score of all 3 raters was used for the final result. Patient characteristics and clinical scores are summarized in the On-line Table.

\section{Imaging}

MR imaging was performed on a 1.5T scanner (Avanto; Siemens, Erlangen, Germany). Most children required either sedation or general anesthesia to obtain adequate images. The imaging protocol included conventional MR imaging sequences (ie, FLAIR, T2WI, DWI) and a 3D-T1-weighted MPRAGE sequence (TR/TE/ TI/flip angle, 1900/2.97/1100 ms/15; matrix, $256 \times 176$; voxel size, $1 \times 1 \times 1 \mathrm{~mm}^{3}$; whole-brain coverage), which was used for the volumetric analysis. Initially, the 3D-T1-weighted MPRAGE sequence was acquired in the axial plane. Due to relatively long observation periods and updates to the software of the scanner, scans were later acquired in the sagittal plane (TR/TE/TI/flip angle, $2280 / 3.64 / 1000 \mathrm{~ms} / 8^{\circ}$; matrix, $256 \times 256$; voxel size, $1 \times$ $\left.1 \times 1 \mathrm{~mm}^{3}\right)$.

\section{Data Evaluation}

We performed brain segmentation by using the FreeSurfer Software Suite (stable Version 5.3.0, May 15, 2013; http://surfer. nmr.mgh.harvard.edu), while disabling the skull-stripping option of FreeSurfer. ${ }^{16}$

Before the segmentation in FreeSurfer, soft tissue and skull were removed by using the Brain Extraction Tool in FSL (BET, Version 2.1, FSL release 5.0, September 2012; http://fsl.fmrib. ox.ac.uk/fsl/fslwiki/BET). ${ }^{17}$ Several options for brain extraction were tested for each dataset, including robust brain center estimation, bias field and neck cleanup, and different fractional intensity threshold " $\mathrm{f}$ " values for the "standard_space_roi" command. We found that an optimal brain extraction, defined as removal of as much extracranial tissue as possible without removing brain tissue, could be achieved by using either robust brain estimation or the standard_space_roi command at $\mathrm{f}=0.15$. For patients with $>1$ study, the longitudinal processing pipeline was used with an intraindividual consistent skull-stripping mask. After segmentation, all datasets were checked visually for major segmentation errors (ie, obvious errors of GM and WM segmentation, inclusion of large amounts of dural venous sinuses or soft tissue). Such studies would have resulted in exclusion from the final analysis. No manual corrections for small segmentation errors were performed to ensure standardized processing.

Of the large number of brain structures segmented by FreeSurfer, the following regions were used for further evaluation based on our hypothesis: supratentorial cortical GM (ie, total cortical GM volume), supratentorial WM (ie, total cortical WM volume), deep GM structures (sum of segmented regions of caudate nucleus, putamen, pallidum, and thalamus), cerebellar GM, cerebellar WM, and lateral ventricles (sum of the lateral ventricles and choroid plexus).

\section{Statistical Analysis}

For the assessment of correlation between different variables, the Pearson correlation coefficient $(r)$ was calculated. A linear correlation was assumed. The first step included the calculation of a correlation of volumetric data and clinical scoring with the age of the patients. Then, a correlation analysis of volumetric data to the overall clinical scoring was performed. Because the clinical score was zero for 15 of 29 data points, the analysis was also performed for clinical scores of $<1$ to demonstrate the ability of the volumetric data to deliver reliable measurements of disease progression, 


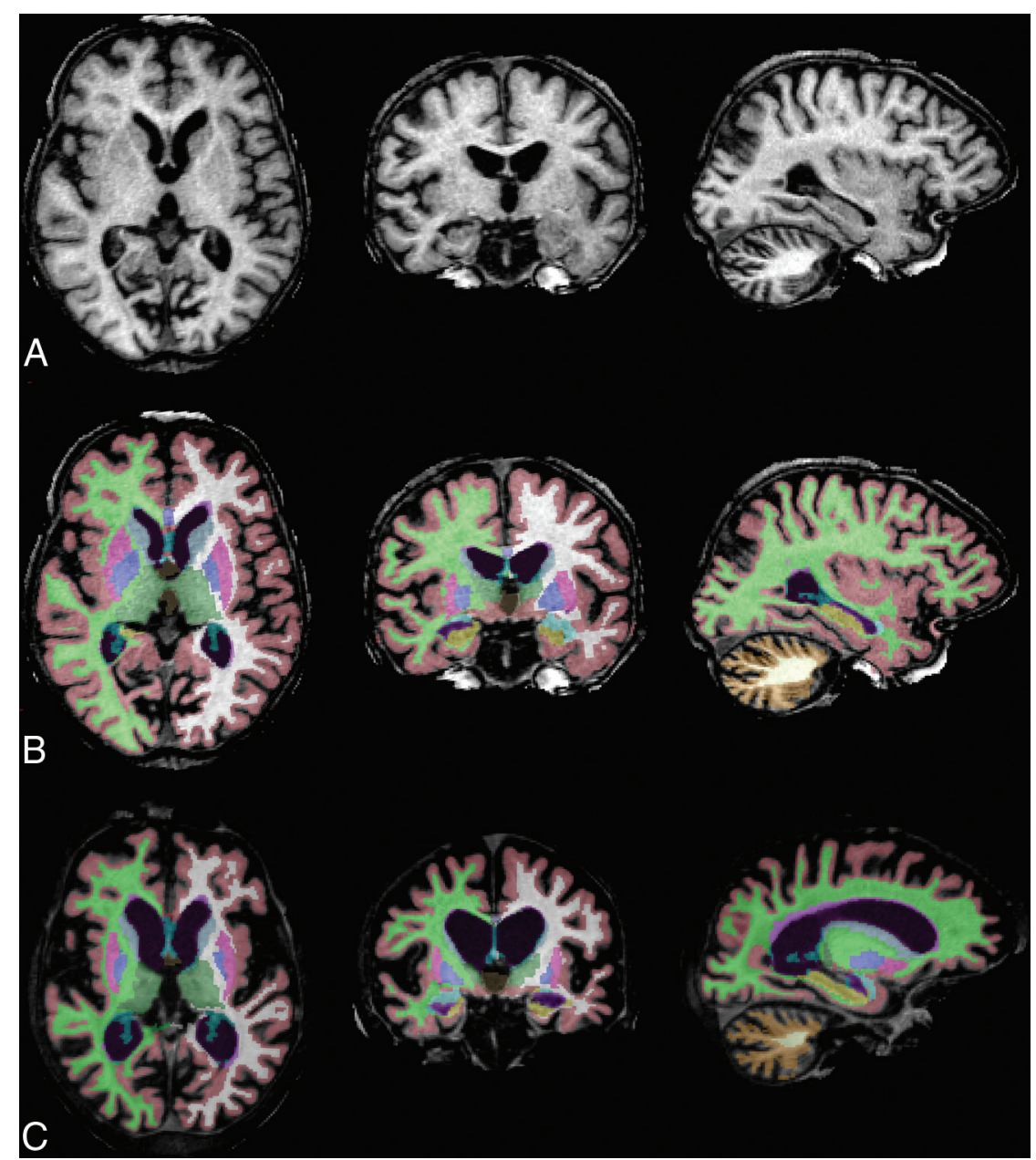

FIG 1. Progressive brain atrophy in CLN2 disease. Baseline 3D-T1-weighted MPRAGE images in axial, coronal, and sagittal planes (left to right) of patient 457-1 (A), segmented brain volumes $(B)$, and segmented brain volumes 4 years later (C).

even at the late stages of the disease. All comparisons were performed at $\alpha=.05$, without adjustment for multiplicity. Patient 467-1 was not included in the statistical analysis due to his unusual clinical presentation (see "Results"). For graphic depiction of the data, we used logarithmic fit functions and confidence intervals and calculated $r^{2}$, again assuming a logarithmic relationship.

\section{RESULTS}

\section{Clinical Data}

Individual clinical disease scores of each patient at the time of baseline and follow-up MR imaging are given in the On-line Table. Most patients had a highly predictable disease course with symptom onset between 2 and 4 years of age and disease progression with complete loss of motor, language, and visual functions by $4-7.5$ years of age. Patient $467-1$ showed a very different disease course with late-onset and slower disease progression.

\section{MR Imaging}

Thirty-one 3D-T1-weighted MPRAGE datasets were available for the 13 patients included in this study. Two datasets could not be processed in FreeSurfer because Talairach transform did not run; this scenario resulted in 29 datasets available for the final data analysis.
Volumes of supratentorial cortical GM, basal ganglia/thalamus, cerebellar GM, and cerebellar WM decreased with age, while the size of the lateral ventricles increased (Figs 1 and 2).

We found that supratentorial cortical GM showed a very uniform decline across the patient cohort. Also, the regions of basal ganglia/thalamus and infratentorial WM showed a strong decline, while the decline was less uniform for cerebellar GM. Supratentorial WM remained relatively stable with time (Fig 2). Patient 467-1, who presented with a juvenile disease onset and first seizures at 8.8 years of age, showed larger brain volumes at 14 years of age compared with all other patients and was, therefore, not included in the statistical analysis.

\section{Correlation of MR Imaging Volumetry with Patient Age and Clinical Scores}

The correlation of volumetric data with patient age was statistically significant for all regions studied but was strongest for supratentorial cortical GM $(r=$ $-0.913, P<.001$ ) (Table 1). Strong correlations were also observed for the basal ganglia/thalamus $(r=-0.832, P<$ $.001)$ and cerebellar WM $(r=-0.830$, $P<.001)$. Correlations of segmented volumes with patient age were significant but less marked for the lateral ventricles $(r=0.606, P=.001)$ and cerebellar GM $(r=-0.659, P<$ $.001)$.

The correlation of volumetric data with clinical scores resulted in high $r$ values for the supratentorial cortical GM $(r=0.862, P<$ $.001)$ and the basal ganglia/thalamus $(r=0.758, P<.001)$, but the correlation was significant for all regions studied. In addition, clinical scores also correlated significantly with patient age $(r=-0.781, P<.001)$.

\section{Correlation of MR Volumetry with Patient Age and Clinical Scores for Scores of Less Than 1}

Generally, clinical motor-language-visual scores of $<1$ were observed in the late disease stages and in older patients $(6-7$ years and older). Looking at MR imaging scans of patients with clinical scores of zero, we found that patient age was significantly correlated with volumes of supratentorial cortical GM, basal ganglia/thalamus, cerebellar GM, and cerebellar WM, but not with the lateral ventricles and supratentorial WM (Table 2).

\section{DISCUSSION}

MR imaging brain volumes of patients with genetically confirmed CLN2 disease showed a marked decline with age for all brain regions studied, while ventricular volumes increased. Segmented 

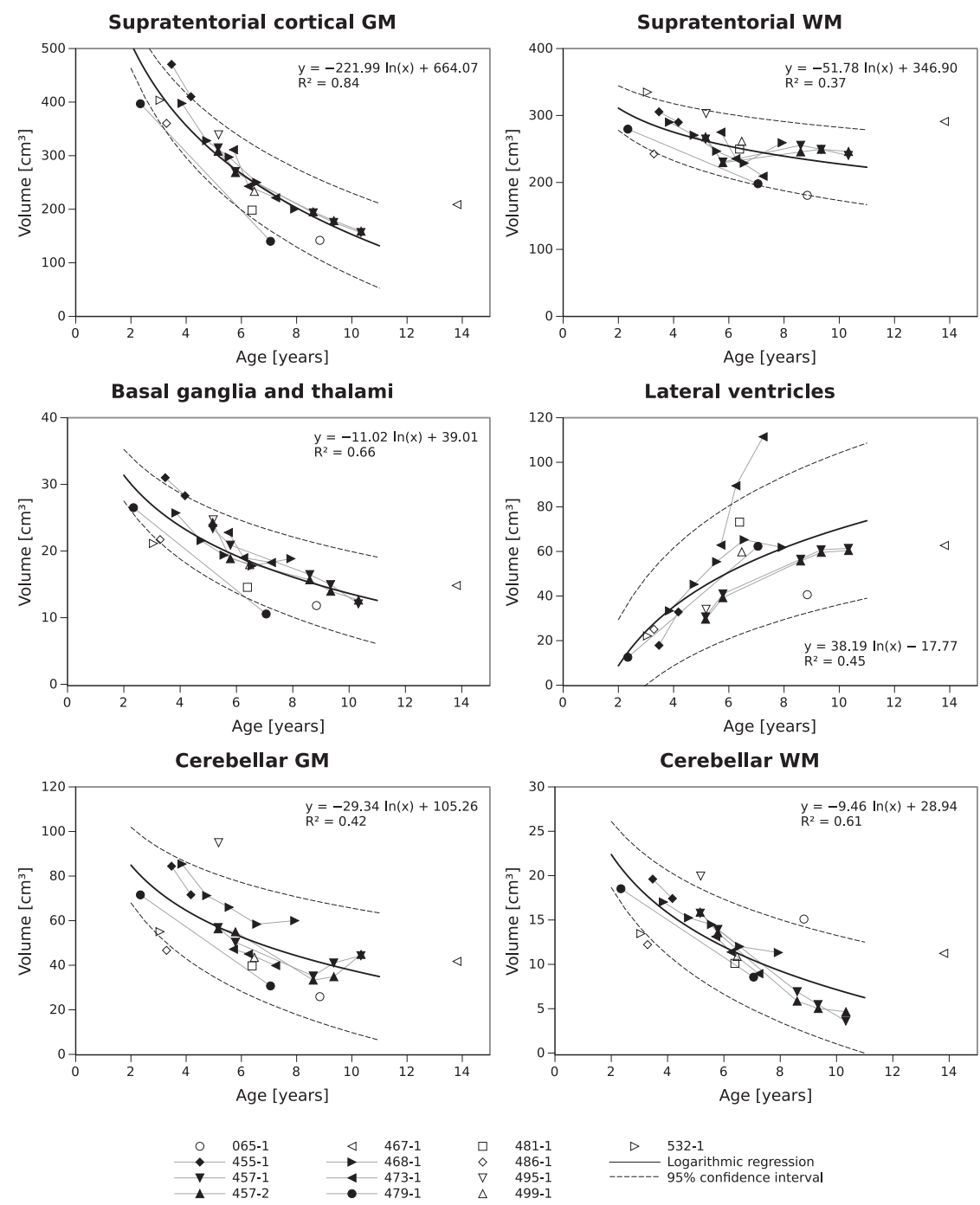

FIG 2. Age dependence of segmented brain volumes, logarithmic fit functions, and confidence intervals.

brain volumes were strongly correlated to patient age and, though to a lesser extent, to a disease-specific clinical score. In line with our hypothesis, we observed the strongest correlation with age and clinical scores for GM regions, more specifically, supratentorial cortical GM and basal ganglia/thalamus. In addition, we correlated brain volumes with the age of the patient during the late stages of the disease when the clinical scoring cannot assess disease progression because the combined motor, language, and visual scores were zero. We found that in those patients, brain volumes were still significantly correlated with age for supratentorial cortical GM, basal ganglia/thalamus, cerebellar GM, and cerebellar WM. A single patient, who presented with a juvenile disease onset, showed much higher brain volumes compared with patients with late-infantile presentation.

The strong correlation of GM volumes to patient age and clinical scores implies that GM regions are more suitable to assess disease progression in CLN2 disease compared with the size of lateral ventricles or supratentorial WM used previously. ${ }^{13,14,18}$ of GM regions studied, the supratentorial cortical GM showed the highest correlation to age and clinical scoring but also a relatively uniform decline across the patient cohort. Therefore, supratento- rial cortical GM appears to be the region most suited for an assessment of disease progression in CLN2 disease. The finding that GM regions showed a more uniform decline compared with $\mathrm{WM}$ regions is also in keeping with histopathologic findings of the underlying neuronal degeneration in CLN2 disease. ${ }^{4,19}$ In addition to cortical regions, neuronal depletion of deep GM regions (ie, the basal ganglia and thalamus) is not well-studied but is reported to be less extensive compared with the cerebral and cerebellar cortices. ${ }^{19}$

In contrast, we observed stronger correlations of brain volumes of the basal ganglia/thalamus with patient age and clinical scores compared with cerebellar GM. This may be related to difficulties in segmenting the cerebellum in patients with very significant brain atrophy. Figure 2 shows that cerebellar GM volumes slightly increased for patients 457-1, 457-2, and 468-1 at the latest follow-up scan. This finding is unexpected with respect to the clinical disease course with progressive loss of motor, language, and visual functions. Therefore, these findings must represent segmentation artifacts, which are most likely due to small cerebellar volumes and tight folding compared with the cerebrum (see Fig $1 B,-C$, column far right). The finding that $\mathrm{MR}$ imaging brain volumes correlated with age, even at disease stages when clinical scores were zero, suggests that MR imaging volumetry provides a quantitative tool to assess disease progression even at late stages of the disease when progression cannot be detected by clinical scoring.

Patient 467-1 was an outlier with a juvenile disease onset at 8 years of age. Patients with CLN2 usually present between 2 and 4 years of age. The different phenotype may be explained by the patient's genetic background (compound heterozygous mutation with c.509-1G $>$ C/c.1439T $>$ G). The c.509-1G $>$ C mutation usually occurs in patients with the classic late-infantile phenotypes, while the c.1439T $>$ G mutation has been reported in patients with early juvenile disease. $^{3,6}$

The most suitable study for a comparison of our data with that of healthy volunteers was published in 2012. ${ }^{20}$ A comparison of brain volumes of our patients for whole-brain GM and WM, whole cerebellum, and ventricles starting at 4.8 years of age revealed distinctively smaller volumes for whole-brain GM, whole-brain WM, and the cerebellum compared with healthy controls. In contrast, the size of the lateral ventricles was increased. These differences were much more pronounced for older patients.

Previously, longitudinal brain atrophy in CLN2 has only been assessed indirectly by using the size of CSF spaces. ${ }^{14} \mathrm{CSF}$ space was 
Table 1: Correlation of brain volumes with patient age and clinical motor-language-visual

\begin{tabular}{|c|c|c|c|c|}
\hline & \multicolumn{2}{|c|}{ Patient Age } & \multicolumn{2}{|c|}{ Clinical Score } \\
\hline & $\begin{array}{l}\text { Correlation } \\
\text { Coefficient } r\end{array}$ & $P$ Value $^{\mathrm{a}}$ & $\begin{array}{l}\text { Correlation } \\
\text { Coefficient } r\end{array}$ & $P$ Value \\
\hline \multicolumn{5}{|l|}{ ROI } \\
\hline Lateral ventricles & 0.606 & .001 & -0.692 & $<.001$ \\
\hline Supratentorial cortical GM & -0.913 & $<.001$ & 0.862 & $<.001$ \\
\hline Supratentorial WM & -0.865 & $<.001$ & 0.735 & $<.001$ \\
\hline Basal ganglia/thalamus & -0.832 & $<.001$ & 0.758 & $<.001$ \\
\hline Cerebellar GM & -0.659 & $<.001$ & 0.609 & .001 \\
\hline Cerebellar WM & -0.830 & $<.001$ & 0.638 & $<.001$ \\
\hline Clinical score & -0.781 & $<.001$ & NA & \\
\hline
\end{tabular}

Note:-NA indicates not applicable.

${ }^{a}$ All $P$ values are statistically significant.

Table 2: Correlation of brain volumes with patient age and clinical motor-language-visual scores for patients with very low clinical scores $(<1)$

\begin{tabular}{lcclcc}
\hline & \multicolumn{2}{c}{ Patient Age } & & \multicolumn{2}{c}{ Clinical Scores $<1$} \\
\cline { 2 - 3 } & $\begin{array}{c}\text { Correlation } \\
\text { Coefficient } \boldsymbol{r}\end{array}$ & $\boldsymbol{P}$ Value & & $\begin{array}{c}\text { Correlation } \\
\text { Coefficient } \boldsymbol{r}\end{array}$ & $\boldsymbol{P}$ Value \\
\hline ROI & -0.068 & .810 & & NA & NA \\
Lateral ventricles & -0.903 & $<.001^{\mathrm{a}}$ & & NA & NA \\
Supratentorial cortical GM & 0.111 & .695 & & NA & NA \\
Supratentorial WM & -0.822 & $<.001^{\mathrm{a}}$ & & NA & NA \\
Basal ganglia/thalamus & -0.548 & $.035^{\mathrm{a}}$ & & NA & NA \\
Cerebellar GM & -0.795 & $<.001^{\mathrm{a}}$ & & NA & NA \\
Cerebellar WM & NA & NA & & NA & NA \\
Clinical score & & & & & \\
\hline
\end{tabular}

Note:-NA indicates not applicable.

a Statistically significant.

inversely correlated with the clinical scoring. However, the correlations of brain volume changes of GM and WM regions in our cohort were stronger; this finding suggests that whole-brain segmentation is more suitable to assess disease progression.

Some limitations of our study need to be addressed. The recruitment of patients and collection of clinical and MR imaging data were initiated in a prospective fashion. However, 3D-T1WI parameters (ie, primary imaging plane, TR, TE, water excitation) of the datasets used in this report varied due to the long observation period and adjustments due to scanner software updates. This may have resulted in a higher variability of segmentation results, especially during the late stages of the disease. Here, optimization of sequence parameters, including the use of multiecho MPRAGE, may improve the segmentation process in patients with severe atrophy. However, the decline of GM volumes was very homogeneous, especially for supratentorial cortical GM. It can, therefore, be concluded that slight changes to the sequence parameters may not strongly affect volumetric outcomes in our patients with CLN2. Possibly, the use of a more standardized dataset may have provided significant correlations to the clinical scores for supratentorial WM regions as well.

\section{CONCLUSIONS}

The MR imaging assessment of brain volumes in patients with genetically confirmed CLN2 disease revealed a very uniform progression of brain atrophy, strongly related to patient age. Brain volumes also correlated with the clinical score, but to a slightly lesser extent. MR imaging volumetry allows the assessment of progressive brain volume loss even during late stages of the disease when the clinical disease score cannot depict any clinical changes. Our data suggest that MR imaging volumetry is an objective and highly sensitive tool to quantitatively describe disease progression and to assess the efficacy of experimental therapies in all stages of CLN2 disease.

\section{ACKNOWLEDGMENTS}

We would like to extend our gratitude to Waltraud Hubert (children's nurse/ study nurse) for her outstanding patient care. We thank all patients and families as well as referring physicians for giving us the opportunity to collect these data.

Disclosures: Miriam Nickel—RELATED: Grant: DEMCHILD* (A Treatment-Oriented Research Project of NCL Disorders as a Major Cause of Dementia in Childhood); UNRELATED: Consultancy: BioMarin, Spark Therapeutics. Jens Fiehler-UNRELATED: Consultancy: Acandis, Medina Medical/Medtronic, Sequent Medical, Stryker; Grants/Grants Pending: MicroVention*; Payment for Lectures (including service on Speakers Bureaus): Boehringer Ingelheim, Covidien/Medtronic, Penumbra; Travel/Accommodations/Meeting Expenses Unrelated to Activities Listed: Medtronic. Igor Nestrasil-UNRELATED: Consultancy: BioMarin, ArmaGen; Grants/ Grants Pending: BioMarin,* Shire,* Genzyme.* Alfried Kohlschütter-UNELATED: Consultancy: BioMarin, Comments: consulting on the natural clinical course of the disease in question and on a clinical trial with an experimental drug; Travel/Accommodations/Meeting Expenses Unrelated to Activities Listed: BioMarin Pharmaceuticals. Angela Schulz-RELATED: Grant: European Commission (FP7 Project DEM-CHILD)*; UNRELATED: Consultancy: BioMarin,* Spark Therapeutics*; Grants/Grants Pending: BioMarin, ${ }^{*}$ Comments: grant to support development of an international DEM-CHILD patient registry. *Money paid to the institution.

\section{REFERENCES}

1. Haltia M, Goebel HH. The neuronal ceroid-lipofuscinoses: a historical introduction. Biochim Biophys Acta 2013;1832:1795-800 CrossRef Medline

2. Warrier V, Vieira M, Mole SE. Genetic basis and phenotypic correlations of the neuronal ceroid lipofusinoses. Biochim Biophys Acta 2013;1832:1827-30 CrossRef Medline

3. Kousi M, Lehesjoki AE, Mole SE. Update of the mutation spectrum and clinical correlations of over 360 mutations in eight genes that underlie the neuronal ceroid lipofuscinoses. Hum Mutat 2012;33: 42-63 CrossRef Medline

4. Jadav RH, Sinha S, Yasha TC, et al. Clinical, electrophysiological, imaging, and ultrastructural description in 68 patients with neuronal ceroid lipofuscinoses and its subtypes. Pediatr Neurol 2014;50:85-95 CrossRef Medline

5. Bessa C, Teixeira CA, Dias A, et al. CLN2/TPP1 deficiency: the novel mutation IVS7-10A $>\mathrm{G}$ causes intron retention and is associated with a mild disease phenotype. Mol Genet Metab 2008;93:66-73 CrossRef Medline

6. Elleder M, Dvoráková L, Stolnaja L, et al. Atypical CLN2 with later onset and prolonged course: a neuropathologic study showing different sensitivity of neuronal subpopulations to TPP1 deficiency. Acta Neuropathol (Berl) 2008;116:119-24 CrossRef Medline

7. Kohan R, Carabelos MN, Xin W, et al. Neuronal ceroid lipofuscinosis type CLN2: a new rationale for the construction of phenotypic subgroups based on a survey of 25 cases in South America. Gene 2013;516:114-21 CrossRef Medline 
8. Schulz A, Kohlschütter A, Mink J, et al. NCL diseases: clinical perspectives. Biochim Biophys Acta 2013;1832:1801-06 CrossRef Medline

9. Paniagua Bravo A, Forkert ND, Schulz A, et al. Quantitative T2 measurements in juvenile and late infantile neuronal ceroid lipofuscinosis. Clin Neuroradiol 2013;23:189-96 CrossRef Medline

10. Dyke JP, Voss HU, Sondhi D, et al. Assessing disease severity in late infantile neuronal ceroid lipofuscinosis using quantitative MR diffusion-weighted imaging. AJNR Am J Neuroradiol 2007;28:1232-36 CrossRef Medline

11. Autti T, Raininko R, Santavuori P, et al. MRI of neuronal ceroid lipofuscinosis, II: postmortem MRI and histopathological study of the brain in 16 cases of neuronal ceroid lipofuscinosis of juvenile or late infantile type. Neuroradiology 1997;39:371-77 CrossRef Medline

12. Löbel U, Sedlacik J, Güllmar D, et al. Diffusion tensor imaging: the normal evolution of ADC, RA, FA, and eigenvalues studied in multiple anatomical regions of the brain. Neuroradiology 2009;51: 253-63 CrossRef Medline

13. Autti TH, Hämäläinen J, Mannerkoski M, et al. JNCL patients show marked brain volume alterations on longitudinal MRI in adolescence. J Neurol 2008;255:1226-30 CrossRef Medline
14. Dyke JP, Sondhi D, Voss HU, et al. Assessment of disease severity in late infantile neuronal ceroid lipofuscinosis using multiparametric MR imaging. AJNR Am J Neuroradiol 2013;34:884-89 CrossRef Medline

15. Steinfeld R, Heim P, von Gregory H, et al. Late infantile neuronal ceroid lipofuscinosis: quantitative description of the clinical course in patients with CLN2 mutations. Am J Med Genet 2002;112:347-54 CrossRef Medline

16. Fischl B. FreeSurfer. Neuroimage 2012;62:774-81 CrossRef Medline

17. Smith SM. Fast robust automated brain extraction. Hum Brain Mapp 2002;17:143-55 CrossRef Medline

18. Worgall S, Kekatpure MV, Heier L, et al. Neurological deterioration in late infantile neuronal ceroid lipofuscinosis. Neurology 2007;69: 521-35 CrossRef Medline

19. Anderson GW, Goebel HH, Simonati A. Human pathology in NCL. Biochim Biophys Acta 2013;1832:1807-26 CrossRef Medline

20. Brain Development Cooperative Group. Total and regional brain volumes in a population-based normative sample from 4 to 18 years: the NIH MRI Study of Normal Brain Development. Cereb Cortex 2012;22:1-12 CrossRef Medline 